\title{
The $q$-Sumudu transform and its certain properties in a generalized $q$-calculus theory
}

\section{Shrideh Khalaf Al-Omari ${ }^{1 *}$ (D)}

Correspondence:

s.k.q.alomari@fet.edu.jo; shridehalomari@bau.edu.jo

${ }^{1}$ Faculty of Engineering Technology, Department of Physics and Basic Sciences, Al-Balqa Applied University, 11134 Amman, Jordan

\section{Springer}

\begin{abstract}
In this paper we consider a generalization to the $q$-calculus theory in the space of $q$-integrable functions. We introduce $q$-delta sequences and develop $q$-convolution products to derive certain $q$-convolution theorem. By using the concept of $q$-delta sequences, we establish various axioms and set up $q$-spaces of generalized functions named $q$-Boehmian spaces. The new assigned spaces of $q$-generalized functions are acceptable and compatible with the classical spaces of the ordinary functions. Consequently, we extend the generalized q-Sumudu transform to the sets of $q$-Boehmian spaces. On top of that, we nominate the canonical $q$-embeddings between the $q$-integrable sets of functions and the $q$-integrable sets of $q$-Boehmians. Furthermore, we address the general properties of the generalized q-Sumudu transform and its inversion formula in some detail.
\end{abstract}

MSC: Primary 54C40; 14E20; secondary 46E25; 20C20

Keywords: $q$-delta sequences; $q$-Sumudu transform; $q$-convolution; $q$-Boehmian; q-calculus

\section{Preliminaries}

The subject of fractional calculus has attained an eminent concern during the past decades due to various applications of this subject in various fields of science and engineering. Recently, an increase of interest in this area has duly been implemented and utilized in the theory of ordinary fractional calculus, optimal control problems, $q$-transform analysis, statistics, mathematical physics, $q$-difference equations, and $q$-integral equations (see, e.g., $[1,2])$. By fixing a real number $q$ such that $0<q<1$, the $q$-derivative of a differentiable function $\vartheta$ is defined by

$$
D_{q} \vartheta(x)=\frac{\vartheta(x)-\vartheta(q x)}{(1-q) x} \quad(x \neq 0) .
$$

The $q$-integrals from 0 to $y$ and from 0 to $\infty$ have been respectively defined by Jackson [3] as follows:

$$
I_{q} \vartheta(y)=\int_{0}^{y} \vartheta(x) d_{q} x=(1-q) y \sum_{n=0}^{\infty} \vartheta\left(y q^{n}\right) q^{n}
$$

(c) The Author(s) 2021. This article is licensed under a Creative Commons Attribution 4.0 International License, which permits use, sharing, adaptation, distribution and reproduction in any medium or format, as long as you give appropriate credit to the original author(s) and the source, provide a link to the Creative Commons licence, and indicate if changes were made. The images or other third party material in this article are included in the article's Creative Commons licence, unless indicated otherwise in a credit line to the material. If material is not included in the article's Creative Commons licence and your intended use is not permitted by statutory regulation or exceeds the permitted use, you will need to obtain permission directly from the copyright holder. To view a copy of this licence, visit http://creativecommons.org/licenses/by/4.0/. 
and

$$
\int_{0}^{\infty} \vartheta(x) d_{q} x=(1-q) \sum_{n=-\infty}^{\infty} \vartheta\left(q^{n}\right) q^{n}
$$

provided the two sums converge absolutely. The $q$-integration by parts has been defined by

$$
\int_{a}^{b} g(x) D_{q} \vartheta(x) d_{q} x=\vartheta(b) g(b)-\vartheta(a) g(a)-\int_{a}^{b} \vartheta(q x) D_{q} g(x) d_{q} x .
$$

Ever after Jackson [3] presented the $q$-integral concept, various $q$-analogues of various types of integral transforms were given in a classical way (see, e.g., [4-19]). Consisting of the notion of regular operators [20], the theory of Boehmians was first introduced by [21] to generalize distributions and regular operators [22] (see, e.g., [21, 23-31]). Boehmians with their abstract nature are equivalence classes of quotients of sequences obtained from an integral domain, where the operations are addition and convolution. Complying with the $q$-calculus concept, we introduce the $q$-Boehmian concept as follows: Let $A$ be a complex linear space and $B$ be a subspace of $A$. Let $\bullet^{q}: A \times B \rightarrow A$ be a binary operation such that, for all $\alpha \in \mathbb{C}, \hat{\delta}, \check{\delta}, \tilde{\delta} \in A$ and $\gamma_{1}, \gamma_{2}, \gamma \in B$, we have $(\hat{\delta}+\check{\delta}) \bullet^{q} \gamma=\hat{\delta} \bullet \bullet^{q} \gamma+\check{\delta} \bullet \bullet^{q} \gamma$, $(\alpha \hat{\delta}) \bullet^{q} \gamma=\alpha(\hat{\delta} \bullet q), \hat{\delta} \bullet^{q}\left(\gamma_{1} \bullet^{q} \gamma_{2}\right)=\left(\hat{\delta} \bullet^{q} \gamma_{1}\right) \bullet^{q} \gamma_{2}, \tilde{\delta}_{n} \bullet{ }^{q} \gamma \rightarrow \tilde{\delta} \bullet^{q} \gamma$ as $\tilde{\delta}_{n} \rightarrow \tilde{\delta}$ in $A$ as $n \rightarrow \infty$ and, for all $\left(x_{n}\right),\left(y_{n}\right) \in \Delta_{q}$, we have $x_{n} \bullet q y_{n} \in \Delta_{q}$, where $\Delta_{q}$ is a collection of sequences in $B$ and $x_{n} \bullet^{q} \tilde{\delta}_{n} \rightarrow \tilde{\delta}$ as $n \rightarrow \infty$ provided $\tilde{\delta}_{n} \rightarrow \tilde{\delta}$ in $A$ as $n \rightarrow \infty$. The name of $q$-Boehmian is proposed to mean the equivalence class $\frac{\tilde{\delta}_{n}}{x_{n}}$ obtained from the equivalence relation

$$
\tilde{\delta}_{n} \bullet^{q} x_{n}=\tilde{\delta}_{m} \bullet^{q} x_{n} \quad(m, n \in \mathbb{N}),
$$

where $\left(\tilde{\delta}_{n}\right) \in A$ and $\left(x_{n}\right) \in \Delta_{q}$. The collection of all $q$-Boehmians is claimed to form a $q$ Boehmian space denoted by $B_{q}$. The linear space $A$ is identified as a subspace of the $q$ Boehmian space $B_{q}$ justified by the identification formula

$$
\check{\delta} \rightarrow \frac{\check{\delta} \bullet^{q} x_{n}}{x_{n}}, \quad \text { where } \check{\delta} \in A \text { and }\left(x_{n}\right) \in \Delta_{q} \text {. }
$$

Two $q$-Boehmians $\frac{\hat{\delta}_{n}}{x_{n}}$ and $\frac{\tilde{\delta}_{n}}{y_{n}}$ are said to be equal in $B_{q}$ if $\hat{\delta}_{n} \bullet^{q} x_{m}=\tilde{\delta}_{m} \bullet q x_{m}, \forall m, n \in \mathbb{N}$. The addition is defined in $B_{q}$ as

$$
\frac{\hat{\delta}_{n}}{x_{n}}+\frac{\tilde{\delta}_{n}}{y_{n}}=\frac{\hat{\delta}_{n} \bullet{ }^{q} y_{n}+\tilde{\delta}_{n} \bullet q x_{n}}{x_{n} \bullet q y_{n}} .
$$

The scalar multiplication is defined in $B_{q}$ as

$$
\alpha \frac{\tilde{\delta}_{n}}{x_{n}}=\frac{\alpha \tilde{\delta}_{n}}{x_{n}} \quad \text { for all } \alpha \in \mathbb{C} \text {. }
$$

For every $\left(x_{n}\right) \in \Delta_{q}$, convergence of type $\delta_{q}, \beta_{n} \stackrel{\delta_{q}}{\rightarrow} \beta$, is defined in $B_{q}$ when

$$
\beta_{n} \bullet{ }^{q} x_{k} \in A, \forall k, n \in \mathbb{N}, \beta \bullet q x_{k} \in A, \forall k \in \mathbb{N},
$$


and for each $k \in \mathbb{N}, \beta_{n} \bullet^{q} x_{k} \rightarrow \beta \bullet \bullet^{q} x_{k}$ as $n \rightarrow \infty$ in $A$, whereas, convergence of type $\Delta_{q}$, $\beta_{n} \stackrel{\Delta_{q}}{\rightarrow} \beta$, is defined in $B_{q}$ if for some $\left(x_{n}\right) \in \Delta_{q},\left(\beta_{n}-\beta\right) \bullet^{q} x_{n} \in A, \forall n \in \mathbb{N}$ and

$$
\left(\beta_{n}-\beta\right) \bullet^{q} x_{n} \rightarrow 0 \quad \text { as } n \rightarrow \infty \text { in } A .
$$

The space of $q$-Boehmians furnished with the current convergence notions emerges to be a complete quasi-normed space. Over the set of functions

$$
A=\left\{\check{\delta}(t): \exists M, \tau_{1}, \tau_{2}>0,|\check{\delta}(t)|<M E_{q}\left(\frac{|t|}{\tau_{j}}\right), t \in(-1)^{j} \times[0, \infty)\right\},
$$

the $q$-analogue of the Sumudu transform of the first type was latterly defined by [8, (1.18)] as follows:

$$
S_{q}\{\check{\delta}(t) ; s\}=\frac{1}{(1-q) s} \int_{0}^{s} E_{q}\left(\frac{q}{s} t\right) \check{\delta}(t) d_{q} t, \quad s \in\left(-\tau_{1}, \tau_{2}\right) .
$$

The properties of $q$-analogue $S_{q}$ of the Sumudu transform including the convergence conditions and its relation with the $q$-Laplace integral transform have been derived by $\mathrm{Al}$ bayrak et al. [8]. Over and above, the authors investigated certain fundamental aspects of the cited integral enfolding linearity, shifting theorems, differentiation, integration, etc. Also an attempt has been made to obtain the convolution theorem in a convergent series type. On the other hand, the authors in [6] provided some applications of the $q$-Sumudu transform to $q$-polynomials, $q$-functions, and $q$-Fox's $H$-functions as well.

The aimed goal of this paper is to discuss the generalized $q$-theory of the $q$-integrable functions in the space $L_{q}^{1}$ and to investigate fundamental properties of the $q$-analogue (1) in the generalized $q$-theory. In Sect. 2, we introduce a concept of $q$-Boehmians and a concept of $q$-delta sequences. We also establish the space $B_{q}^{1}$ of $q$-Boehmians. In Sect. 3, we establish the second space $B_{q}^{2}$ of $q$-Boehmians. In Sect. 4, we introduce the generalized $q$-Sumudu transform and discuss several properties. In Sect. 5 , we provide a conclusion part.

\section{The space $B_{q}^{1}$}

Denote by $L_{q}^{1}$ the space of all $q$-integrable functions $\check{\delta}$ on $\mathbb{R}_{+}$defined by

$$
\|\check{\delta}\|_{L_{q}^{1}\left(\mathbb{R}_{+}\right)}=\frac{1}{1-q} \int_{0}^{\infty}|\check{\delta}(x)| d_{q} x<\infty
$$

whose comparable definition in a series expression formula is given as $\sum_{-\infty}^{\infty} q^{n} \check{\delta}\left(q^{n}\right)$, provided the series converges absolutely. Denote by $D_{q}$ the $q$-space of all test functions of compact supports on $\mathbb{R}_{+}$, i.e.,

$$
D_{q}=\left\{\check{\delta} \in C^{\infty}(\mathbb{R}): \sup _{0<x<\infty}\left|D_{q} \check{\delta}(x)\right|<\infty\right\} .
$$

Denote by $\Delta_{q}$ the set of all sequences from $D_{q}$ such that $\Delta_{q}^{1}-\Delta_{q}^{3}$ satisfy

$$
\Delta_{q}^{1}: \int_{0}^{\infty}\left|x_{n}(x)\right| d_{q} x=1, \quad \forall n \in \mathbb{N} .
$$




$$
\begin{aligned}
& \Delta_{q}^{2}:\left|x_{n}(x)\right|<M, \quad M>0, M \in \mathbb{R}_{+} . \\
& \Delta_{q}^{3}: \operatorname{supp}\left(x_{n}\right) \subseteq\left(0, b_{n}\right), b_{n} \rightarrow 0 \quad \text { as } n \rightarrow \infty, \forall n \in \mathbb{N} .
\end{aligned}
$$

Between two integrable functions $\check{\delta}$ and $\hat{\delta}$ in $L_{q}^{1}$, we denote by $*^{q}$ the $q$-convolution product defined by

$$
\left(\check{\delta} *^{q} \hat{\delta}\right)(x)=\int_{0}^{x} \check{\delta}(x-t) \hat{\delta}(t) d_{q} t
$$

provided the right-hand side integral exists for every real number $x>0$. It is clear from the context that $\check{\delta} *^{q} \hat{\delta} \in L_{q}^{1}$ for all $\check{\delta}$ and $\hat{\delta}$ in $L_{q}^{1}$. Following Belgacem [32], the $q$-convolution theorem of the $q$-Sumudu transform of the convolution $\check{\delta} *^{q} \hat{\delta}$ can be easily established by using $[21,(2.1)]$ (see, also [11]) as follows:

$$
S_{q}\left(\check{\delta} *^{q} \hat{\delta}\right)(x)=x S_{q} \check{\delta}(x) S_{q} \hat{\delta}(x) .
$$

An imperative result for categorizing the $q$-delta sequences may be introduced as follows.

Lemma 1 Let $\left(x_{n}\right)$ and $\left(y_{n}\right)$ be arbitrary in $\Delta_{q}$. Then the sequence $\left(x_{n} *^{q} y_{n}\right)$ is a q-delta sequence in $\Delta_{q}$.

Proof To establish this lemma, we examine that $\Delta_{q}^{1}, \Delta_{q}^{2}$, and $\Delta_{q}^{3}$ satisfy for $\left(x_{n} *^{q} y_{n}\right)$. To examine the correctness of $\Delta_{q}^{1}$, we use integral Eq. (3) to write

$$
\int_{0}^{\infty}\left(x_{n} *^{q} y_{n}\right)(x) d_{q} x=\int_{0}^{\infty}\left(\int_{0}^{x} x_{n}(x-t) y_{n}(t) d_{q} t\right) d_{q} x
$$

Now we change the order of integration to have

$$
\int_{0}^{\infty}\left(x_{n} *^{q} y_{n}\right)(x) d_{q} x=\int_{0}^{\infty} \int_{t}^{\infty} x_{n}(x-t) y_{n}(t) d_{q} t d_{q} x
$$

By changing the variables in the inner integral, i.e., substituting the change of variables $x-t=y$, hence $d_{q} x=d_{q} y$, the above equation reveals

$$
\int_{0}^{\infty}\left(x_{n} *^{q} y_{n}\right)(x) d_{q} x=\int_{0}^{\infty} y_{n}(t) x_{n}(y) d_{q} t d_{q} y=\int_{0}^{\infty} y_{n}(t) d_{q} t \int_{0}^{\infty} x_{n}(y) d_{q} y=1 .
$$

This proves $\Delta_{q}^{1}$. The proof of $\Delta_{q}^{2}$ follows from the fact that $\left|x_{n} *^{q} y_{n}\right| \leq\left|x_{n}\right|\left|y_{n}\right|$. The proof of $\Delta_{q}^{3}$ follows from the fact that $\operatorname{supp}\left(x_{n} *^{q} y_{n}\right) \subset \operatorname{supp}\left(x_{n}\right)+\operatorname{supp}\left(y_{n}\right)$ for $\left(x_{n}\right),\left(y_{n}\right) \in \Delta_{q}$. This completes the proof of the lemma.

Hence, Lemma 1 shows that every sequence in $\Delta_{q}$ forms an appropriate $q$-delta sequence.

Lemma 2 Let $\check{\delta}$ and $\hat{\delta}$ be in $L_{q}^{1}$. Then, for every $\gamma, \hat{\gamma} \in D_{q}$ and $\alpha \in \mathbb{C}$, the following assertions are valid:
(i) $\gamma *^{q} \hat{\gamma}=\hat{\gamma} *^{q} \gamma$,
(ii) $(\check{\delta}+\hat{\delta}) *^{q} \gamma=\check{\delta} *^{q} \gamma+\hat{\delta} * \gamma$,
(iii) $(\alpha \check{\delta}) *^{q} \gamma=\alpha\left(\check{\delta} *^{q} \gamma\right)$,
(iv) $\check{\delta} *^{q}\left(\gamma *^{q} \hat{\gamma}\right)=\left(\check{\delta} *^{q} \gamma\right) *^{q} \hat{\gamma}$. 
Proof (i) By using the definition of the convolution product $*^{q}$ given by (3) and applying the change of variables $y=x-t$, hence $d_{q} y=-d_{q} t$, we get

$$
\begin{aligned}
\left(\gamma *^{q} \hat{\psi}\right)(x) & =\int_{0}^{x} \gamma(x-t) \hat{\gamma}(t) d_{q} t \\
& =\int_{x}^{0} \gamma(y) \hat{\gamma}(x-y)(-1) d_{q} y \\
& =\int_{0}^{x} \hat{\gamma}(x-y) \gamma(y) d_{q} y .
\end{aligned}
$$

That is,

$$
\left(\gamma *^{q} \hat{\psi}\right)(x)=\left(\hat{\gamma} *^{q} \gamma\right)(x)
$$

Proof of (ii) and (iii) follows from simple integral calculus. The proof of (iv) follows from a similar argument to that of (i). This completes the proof of the lemma.

Lemma 3 Let $\check{\delta}$ and $\left(\check{\delta}_{n}\right)$ be sequences of integrable functions in the space $L_{q}^{1}$ such that $\check{\delta}_{n} \rightarrow \check{\delta}$ as $n \rightarrow \infty$. Then we have

$$
\check{\delta}_{n} *^{q} \gamma \rightarrow \check{\delta} *^{q} \gamma \quad \text { as } n \rightarrow \infty
$$

for every $\gamma \in D_{q}$.

The proof of this lemma follows from simple integration. Hence, we delete the details.

Finally, we have to establish the following lemma.

Lemma 4 Let $\check{\delta}$ and $\hat{\delta}$ be arbitrary functions in $L_{q}^{1}$ and $\left(x_{n}\right)$ be in $\Delta_{q}$ such that $\check{\delta} *^{q} x_{n}=$ $\hat{\delta} *^{q} x_{n}$. Then we have $\check{\delta}=\hat{\delta}$ in $L_{q}^{1}$ for every $n \in \mathbb{N}$.

Proof The proof of this lemma follows from Eq. (2) and Lemma 3. Thus, we omit the details.

Lemma 5 Let $\check{\delta}$ be an integrable function in $L_{q}^{1}$, then, for every $\left(x_{n}\right)$ in $\Delta_{q}$, we have $\check{\delta} *^{q} x_{n} \rightarrow$ $\check{\delta}$ as $n \rightarrow \infty$.

Proof By the assumption that $\Delta_{q}^{1}$ and $\Delta_{q}^{3}$ hold for $\left(x_{n}\right)$ and allowing the support of $x_{n}$ to be included in the interval $\left(0, b_{n}\right)$, for some real numbers $b_{n}, n \in \mathbb{N}$, we obtain

$$
\begin{aligned}
\left\|\check{\delta} *^{q} x_{n}-\check{\delta}\right\|_{L_{q}^{1}\left(\mathbb{R}_{+}\right)} & =\frac{1}{1-q} \int_{0}^{\infty}\left|\left(\check{\delta} *^{q} x_{n}\right)(x)-\check{\delta}(x)\right| d_{q} x \\
& \leq \frac{1}{1-q} \int_{0}^{\infty} \int_{0}^{x}|\check{\delta}(x-t)-\check{\delta}(x)|\left|x_{n}(t)\right| d_{q} t d_{q} x \\
& =\frac{1}{1-q} \int_{0}^{\infty} \int_{0}^{b_{n}}|\check{\delta}(x-t)-\check{\delta}(x)|\left|x_{n}(t)\right| d_{q} t d_{q} x .
\end{aligned}
$$


Therefore, we have

$$
\begin{aligned}
\left\|\check{\delta} *^{q} x_{n}-\check{\delta}\right\|_{L_{q}^{1}\left(\mathbb{R}_{+}\right)} \leq & \frac{1}{1-q} \int_{0}^{\infty} \int_{0}^{b_{n}}|\check{\delta}(x-t)|\left|x_{n}(t)\right| d_{q} t d_{q} x \\
& +\frac{1}{1-q} \int_{0}^{\infty} \int_{0}^{b_{n}}|\check{\delta}(x)|\left|x_{n}(t)\right| d_{q} t d_{q} x .
\end{aligned}
$$

Since $\check{\delta} \in L_{q}^{1}$, the above equation can be developed to give

$$
\left\|\check{\delta} *^{q} x_{n}-\check{\delta}\right\|_{L_{q}^{1}\left(\mathbb{R}_{+}\right)} \leq A \frac{1}{1-q} \int_{0}^{b_{n}}\left|x_{n}(t)\right| d_{q} t+A \frac{1}{1-q} \int_{0}^{b_{n}}\left|x_{n}(t)\right| d_{q} t
$$

for some constant $A$. Hence, by applying $\Delta_{q}^{2}$ and integrating, from Eq. (7) we get

$$
\left\|\check{\delta} *^{q} x_{n}-\check{\delta}\right\|_{L_{q}^{1}\left(\mathbb{R}_{+}\right)} \leq A M b_{n}+B M b_{n} \rightarrow 0
$$

as $n \rightarrow \infty$, where $M$ is a certain positive constant. Hence the proof of this lemma is completed.

Therefore, the space $B_{q}^{1}$ is defined with the sets $\left(L_{q}^{1}, *^{q}\right),\left(D_{q}, *^{q}\right)$, and $\Delta_{q}$. The canonical $q$-embedding of the space $L_{q}^{1}$ in the space $B_{q}^{1}$ is given as

$$
\check{\delta} \rightarrow \frac{\check{\delta} *^{q} x_{n}}{x_{n}} .
$$

Therefore, every $\check{\delta}$ in $L_{q}^{1}$ can be identified in $B_{q}^{1}$ as $\frac{\check{\delta} * q_{x_{n}}}{x_{n}}$. In addition, scalar multiplication, differentiation, $\Delta_{q}$ convergence, and $\delta_{q}$ convergence are defined in a natural way. If $\left(\check{\delta}_{n}\right) \in$ $L_{q}^{1}$ and $\left(x_{n}\right) \in \Delta_{q}$, then the pair $\left(\check{\delta}_{n}, x_{n}\right)$ (or $\left.\frac{\check{\delta}_{n}}{x_{n}}\right)$ is said to be a quotient of sequences if $\check{\delta}_{n} *^{q}$ $x_{m}=\check{\delta}_{m} *{ }^{q} x_{n}, \forall n, m \in \mathbb{N}$. Therefore, if $\frac{\check{\delta}_{n}}{x_{n}}$ and $\frac{\hat{\delta}_{n}}{y_{n}}$ are quotients of sequences and $\check{\delta} \in L_{q}^{1}$, then it is easy to see that

$$
\frac{\check{\delta} *^{q} x_{n}}{x_{n}} \text { and } \frac{\check{\delta}_{n} *^{q} x_{n}+\hat{\delta}_{n} *^{q} x_{n}}{x_{n} *^{q} y_{n}}
$$

are also quotients of sequences. Further, the following equivalence relations can be easily checked:

$$
\frac{\check{\delta}_{n}}{x_{n} *^{q} \check{\delta}} \sim \frac{\check{\delta}_{n} *^{q} \check{\delta}}{x_{n}} \text { and } \frac{\check{\delta}_{n}}{x_{n} *^{q} \hat{\delta}_{n}} \sim \frac{\check{\delta}_{n} *^{q} \hat{\delta}_{n}}{x_{n}}
$$

Two quotients of sequences $\frac{\check{\delta}_{n}}{x_{n}}$ and $\frac{\hat{\delta}_{n}}{y_{n}}$ are said to be equivalent if $\check{\delta}_{n} *^{q} y_{m}=\hat{\delta}_{m} *^{q} x_{n}$, $\forall n, m \in \mathbb{N}$. The equivalent class $\breve{w}_{B}=\left(\frac{\check{\delta}_{n}}{x_{n}}\right)$ of quotients of sequences containing $\frac{\check{\delta}_{n}}{x_{n}}$ is said to be a $q$-Boehmian. The space of such $q$-Boehmians is denoted by $B_{q}^{1}$.

For two $q$-Boehmians $\breve{w}_{B}=\frac{\check{\delta}_{n}}{x_{n}}$ and $\breve{z}_{B}=\frac{\hat{\delta}_{n}}{y_{n}}$ in $B_{q}^{1}$, the following are well defined on $B_{q}^{1}$ :
(i) $\breve{w}_{B}+\breve{z}_{B}=\frac{\check{\delta}_{n} *^{q} x_{n}+\hat{\delta}_{n} *^{q} \delta_{n}}{x_{n} *^{q} y_{n}}$,
(ii) $\beta \breve{w}_{B}=\frac{\beta \check{\delta}_{n}}{x_{n}}$, 
(iii) $\breve{w}_{B} *^{q} \breve{z}_{B}=\frac{\check{\delta}_{n} *^{q} \hat{\delta}_{n}}{x_{n} *^{q} y_{n}}, \quad$ (iv) $D^{k} \breve{w}_{B}=\frac{D^{k} \check{\delta}_{n}}{x_{n}}, \quad$ and $\quad$ (v) $\breve{w}_{B} *^{q} \check{\delta}=\frac{\check{\delta}_{n} *^{q} \check{\delta}}{x_{n}}$,

where $k \in \mathbb{R}, \beta \in \mathbb{C}, D^{k} \breve{w}_{B}$ is the $k$ th derivative of $\breve{w}_{B}$ and $\check{\delta} \in L_{q}^{1}$.

Definition 6 For $n=1,2,3, \ldots$ and $\breve{w}_{B, n}, \breve{w}_{B} \in B_{q}^{1}$, the sequence $\left(\breve{w}_{B, n}\right)$ is said to be $\delta_{q}$ convergent to $\breve{w}_{B}$, denoted by $\delta_{q}-\lim _{n \rightarrow \infty} \breve{w}_{B, n}=\breve{w}_{B}$, provided there can be found a delta sequence $\left(x_{n}\right)$ such that

(a) $\left(\breve{w}_{B, n} *^{q} x_{k}\right),\left(\breve{w}_{B} *^{q} x_{k}\right)$ in $L_{q}^{1}$ for all $n, k \in \mathbb{N}$;

(b) $\lim _{n \rightarrow \infty} \breve{w}_{B, n} *^{q} x_{k}=\breve{w}_{B} *^{q} x_{k}$ in $L_{q}^{1}$ for every $k \in \mathbb{N}$.

Definition 7 For $n=1,2,3, \ldots$ and $\breve{w}_{B, n}, \breve{w}_{B} \in B_{q}^{1}$, the sequence $\left(\breve{w}_{B, n}\right)$ is said to be $\Delta_{q}$ convergent to $\breve{w}_{B}$, denoted by $\Delta_{q}-\lim _{n \rightarrow \infty} \breve{w}_{B, n}=\breve{w}_{B}$, provided there can be found a delta sequence $\left(x_{n}\right)$ such that

(a) $\left(\breve{w}_{B, n}-\breve{w}_{B}\right) *^{q} x_{n} \in L_{q}^{1}(\forall n \in \mathbb{N})$;

(b) $\lim _{n \rightarrow \infty}\left(\breve{w}_{B, n}-\breve{w}_{B}\right) *^{q} x_{n}=0$ in $L_{q}^{1}$.

Remark 8 Let $\check{\delta} \in L_{q}^{1}$ and $\left(x_{n}\right) \in \Delta_{q}$ be fixed. Then the mapping

$$
\check{\delta} \rightarrow \breve{w}_{B}
$$

where $\breve{w}_{B}=\frac{\check{\delta} * q x_{n}}{x_{n}}$ is an injective mapping from $L_{q}^{1}$ into $B_{q}^{1}$.

Therefore, it can be easily checked that $L_{q}^{1}$ may be identified as a subspace of $B_{q}^{1}$.

Remark 9 Let $\left(x_{n}\right) \in \Delta_{q}$. Then, if $\check{\delta}_{n} \rightarrow \check{\delta}$ in $L_{q}^{1}$ as $n \rightarrow \infty$, then for all $k \in \mathbb{N}$,

$$
\check{\delta}_{n} *^{q} x_{k} \rightarrow \check{\delta} *^{q} x_{k}
$$

as $n \rightarrow \infty$. That is, $\breve{w}_{B, n} \rightarrow \breve{w}_{B}$ in $B_{q}^{1}$ as $n \rightarrow \infty$.

The above remark states the following.

Theorem 10 The mapping $\check{\delta} \rightarrow \breve{w}_{B}, \breve{w}_{B}=\frac{\check{\delta} * q_{x_{n}}}{x_{n}}$, is a continuous q-embedding of the space $L_{q}^{1}$ into the space $B_{q}^{1}$.

\section{The space $B_{q}^{2}$}

In this section we provide the basic essentials that we need in defining the space $B_{q}^{2}$ of $q$ Boehmians, with the set $\left(L_{q}^{1}, \bullet^{q}\right)$, the subset $\left(D_{q}^{S_{q}}, *^{q}\right)$, the set of delta sequences $\left(\Delta_{q}^{S_{q}}, *^{q}\right)$, the convolution products $*^{q}$, and the operation $\bullet$; see Eq. (9). To establish the space $B_{q}^{2}$, let us define the following convolution product.

Definition 11 Let $\check{\delta}$ and $\hat{\delta}$ be integrable functions in $L_{q}^{1}$. Between $\check{\delta}$ and $\hat{\delta}$ we define the $q$-product $\bullet q$ as follows:

$$
(\check{\delta} \bullet q \hat{\delta})(x)=x S_{q} \check{\delta}(x) \hat{\delta}(x)
$$

where $S_{q} \check{\delta}$ is the $q$-Sumudu transform of $\check{\delta}$. 
We have the following assertion.

Theorem 12 Let $\check{\delta}$ be an integrable function in $L_{q}^{1}$, then $\check{\delta} \bullet^{q} \psi \in L_{q}^{1}$ for all $\psi \in D_{q}^{S_{q}}$.

Proof Let $\check{\delta} \in L_{q}^{1}$ and $\psi=S_{q} \gamma$ for some $\gamma \in D_{q}$. Then, by the definition of the space $L_{q}^{1}$ and the product $\bullet q$, we write

$$
\left\|\check{\delta} \bullet^{q} \psi\right\|_{L_{q}^{1}\left(\mathbb{R}_{+}\right)}=\frac{1}{1-q} \int_{0}^{\infty}|(\check{\delta} \bullet q)(x)| d_{q} x=\frac{1}{1-q} \int_{0}^{\infty} x S_{q} \check{\delta}(x) \psi(x) d_{q} x .
$$

Hence, we have

$$
\left\|\check{\delta} \bullet^{q} \psi\right\|_{L_{q}^{1}\left(\mathbb{R}_{+}\right)} \leq \frac{1}{1-q} \int_{0}^{\infty} x S_{q} \check{\delta}(x) S_{q}(\gamma)(x) d_{q} x .
$$

Therefore, if $[a, b]$ denotes a closed interval containing the support of $\psi$, then the hypothesis that $\check{\delta} \in L_{q}^{1}$ yields

$$
\begin{aligned}
\left\|\check{\delta} \bullet^{q} \psi\right\|_{L_{q}^{1}\left(\mathbb{R}_{+}\right)} & =\frac{1}{1-q} \int_{0}^{\infty}\left|\left(\check{\delta} \bullet^{q} \psi\right)(x)\right| d_{q} x \\
& \leq M \frac{1}{1-q} \int_{a}^{b}\left|x S_{q} \gamma(x)\right| d_{q} x \\
& =M \frac{1}{1-q} \int_{a}^{b}|x \psi(x)| d_{q} x \\
& <\infty
\end{aligned}
$$

for every $\psi \in D_{q}^{S_{q}}, M$ being a positive constant.

This establishes the theorem.

Now, we reciprocate the product $\bullet q$ as follows.

Theorem 13 Let $\check{\delta}$ be an integrable function in $L_{q}^{1}$, then $\check{\delta} \bullet^{q}\left(\psi \bullet \bullet^{q} \hat{\psi}\right)=\left(\check{\delta} \bullet^{q} \psi\right) \bullet^{q} \hat{\psi}$ for all $\psi, \hat{\psi} \in D_{q}^{S_{q}}$.

Proof Let $\psi=S_{q} \gamma, \hat{\psi}=S_{q} \hat{\gamma}, \gamma, \hat{\gamma} \in D_{q}$. Then, by the concept of $q$-convolution $\bullet^{q}$ and Eq. (3), we get

$$
\left(\check{\delta} \bullet^{q}\left(\psi \bullet^{q} \hat{\psi}\right)\right)(x)=x\left(S_{q} \check{\delta}\right)(x)\left(S_{q} \gamma \bullet^{q} S_{q} \hat{\gamma}\right)(x)=x S_{q} \check{\delta}(x) x S_{q} \gamma(x) S_{q} \hat{\gamma}(x)
$$

Rearranging Eq. (11) and again the concept of $q$-convolution $\bullet^{q}$ gives

$$
\begin{aligned}
\left(\check{\delta} \bullet^{q}\left(\psi \bullet^{q} \hat{\psi}\right)\right)(x) & =x\left(x S_{q} \check{\delta}(x) x S_{q} \gamma(x)\right)\left(S_{q} \hat{\gamma}(x)\right) \\
& =x\left(\check{\delta} \bullet^{q} \psi\right) S_{q}(\hat{\gamma})(x) \\
& =\left(\left(\check{\delta} \bullet^{q} \psi\right) \bullet^{q} \hat{\psi}\right)(x) .
\end{aligned}
$$

Hence the proof of the theorem follows. 
Theorem 14 (i) Let $\check{\delta}$ and $\hat{\delta}$ be integrable functions in $L_{q}^{1}$, then $(\check{\delta}+\hat{\delta}) \bullet q \psi=\check{\delta} \bullet q \psi+\hat{\delta} \bullet^{q} \psi$ for all $\psi \in D_{q}^{S_{q}}$.

(ii) Let $\check{\delta}$ be an integrable function in $L_{q}^{1}$, then $(\alpha \check{\delta} \bullet q)=\alpha\left(\check{\delta} \bullet^{q} \psi\right)$ for all $\psi \in D_{q}^{S_{q}}, \alpha \in \mathbb{C}$.

Proof (i) Let $\psi \in D_{q}^{S_{q}}, \psi \in D_{q}^{S_{q}}, \psi=S_{q} \gamma, \gamma \in D_{q}$. Then, by the definition of $\bullet q$ and Eq. (11), we write

$$
\begin{aligned}
\left((\check{\delta}+\hat{\delta}) \bullet^{q} \psi\right)(x) & =x S_{q}(\check{\delta}+\hat{\delta})(x) \psi(x) \\
& =x S_{q} \check{\delta}(x) S_{q} \gamma(x)+x S_{q} \hat{\delta}(x) S_{q} \gamma(x) \\
& =\left(\check{\delta} \bullet^{q} \psi\right)(x)+\left(\hat{\delta} \bullet^{q} \psi\right)(x) .
\end{aligned}
$$

The proof of the second part is similar. The proof of this theorem is therefore completed.

Theorem 15 Let $\check{\delta}, \hat{\delta}$ and $\left(\hat{\delta}_{n}\right)$ be integrable in $L_{q}^{1}$. Then the following hold.

(i) If $\hat{\delta}_{n} \rightarrow \check{\delta}$ in $L_{q}^{1}$ as $n \rightarrow \infty$, then $\hat{\delta}_{n} \bullet^{q} \psi \rightarrow \check{\delta} \bullet q$ for all $\psi \in D_{q}^{S_{q}}, \psi=S_{q} \gamma, \gamma \in D_{q}$ as $n \rightarrow \infty$.

(ii) If $\check{\delta} \bullet^{q} x_{n}=\hat{\delta} \bullet^{q} z_{n}$, then $\check{\delta}=\hat{\delta}$ in $L_{q}^{1}$ for all $\left(z_{n}\right) \in \Delta_{q}^{S_{q}}, z_{n}=S_{q} x_{n}, n \in \mathbb{N}$.

(iii) $\delta \bullet q x_{n} \rightarrow 0$ in $L_{q}^{1}$ for all $\left(x_{n}\right) \in \Delta_{q}^{S_{q}}$ as $n \rightarrow \infty$.

Proof (i) Let $\check{\delta}$, $\hat{\delta}$ and $\left(\hat{\delta}_{n}\right)$ be integrable in $L_{q}^{1}$ and $\psi \in D_{q}^{S_{q}}, \psi=S_{q} \gamma, \gamma \in D_{q}$, such that $\hat{\delta}_{n} \rightarrow \hat{\delta}$ in $L_{q}^{1}$ as $n \rightarrow \infty$. Then we have

$$
\left(\hat{\delta}_{n} \bullet q\right)(x)=x S_{q} \hat{\delta}_{n}(x) S_{q} \gamma(x) \rightarrow x S_{q} \hat{\delta}(x) S_{q} \gamma(x)=x S_{q} \hat{\delta}(x) \psi(x)=\check{\delta} \bullet q \text { as } n \rightarrow \infty \text {. }
$$

Proof (ii) Let $\check{\delta}$ and $\hat{\delta}$ be integrable functions in $L_{q}^{1}$ and $\left(z_{n}\right) \in \Delta_{q}^{S_{q}}, z_{n}=S_{q} x_{n}, n \in \mathbb{N}$ such that $\check{\delta} \bullet{ }^{q} z_{n}=\hat{\delta} \bullet q z_{n}$. Then $x S_{q} \check{\delta}(x) S_{q} x_{n}(x)-x S_{q} \hat{\delta}(x) S_{q}\left(x_{n}\right)(x)=0$ for all $x \in L_{q}^{1}$. Hence, we have

$$
x S_{q} x_{n}(x) S_{q}(\check{\delta}-\hat{\delta})(x)=0
$$

for all $x \in L_{q}^{1}$. Therefore, e infers that $\check{\delta}(x)=\hat{\delta}(x)$ for all $x \in L_{q}^{1}$. The proof of (iii) is analogous. Hence the proof is completed.

If $\left(\check{\delta}_{n}\right) \in L_{q}^{1}$ and $\left(z_{n}\right) \in \Delta_{q}^{S_{q}}, z_{n}=S_{q} x_{n}, n \in \mathbb{N}$, then the pair $\left(\check{\delta}_{n}, z_{n}\right)$ (or $\left.\frac{\check{\delta}_{n}}{z_{n}}\right)$ is said to be a quotient of sequences if $\check{\delta}_{n} \bullet q z_{m}=\check{\delta}_{m} \bullet q z_{n}, \forall n, m \in \mathbb{N}$. Therefore, if $\frac{\check{\delta}_{n}}{z_{n}}$ and $\frac{\hat{\delta}_{n}}{y_{n}}$ are quotients of sequences and $\check{\delta} \in L_{q}^{1}$, then it is easy to see that

$$
\frac{\check{\delta} \bullet^{q} z_{n}}{z_{n}} \text { and } \frac{\check{\delta}_{n} \bullet^{q} z_{n}+\hat{\delta}_{n} \bullet^{q} z_{n}}{z_{n} \bullet^{q} y_{n}}
$$

are quotients of sequences. Further, we can easily check the following equivalence relations:

$$
\frac{\check{\delta}_{n}}{z_{n} \bullet q \check{\delta}} \sim \frac{\check{\delta}_{n} \bullet q \check{\delta}}{z_{n}} \text { and } \frac{\check{\delta}_{n}}{z_{n} \bullet q \hat{\delta}_{n}} \sim \frac{\check{\delta}_{n} \bullet q \hat{\delta}_{n}}{z_{n}} .
$$


Two quotients of sequences $\frac{\check{\delta}_{n}}{z_{n}}$ and $\frac{\hat{\delta}_{n}}{y_{n}}$ are said to be equivalent if $\check{\delta}_{n} \bullet q y_{m}=\hat{\delta}_{m} \bullet q z_{n}$, $\forall n, m \in \mathbb{N}$. The equivalent class $\breve{w}_{B}=\frac{\check{\delta}_{n}}{z_{n}}$ of quotients of sequences containing $\frac{\check{\delta}_{n}}{z_{n}}$ is said to be a $q$-Boehmian. The space of such $q$-Boehmians is denoted by $B_{q}^{2}$. For two $q$-Boehmians $\breve{w}_{B}=\frac{\check{\delta}_{n}}{z_{n}}$ and $\breve{z}_{B}=\frac{\hat{\delta}_{n}}{y_{n}}$ in $B_{q}^{2}$, the following are well defined on $B_{q}^{2}$ :
(i) $\breve{w}_{B}+\breve{z}_{B}=\frac{\check{\delta}_{n} \bullet^{q} z_{n}+\hat{\delta}_{n} \bullet^{q} z_{n}}{z_{n} \bullet^{q} y_{n}}$
(ii) $\beta \breve{w}_{B}=\frac{\beta \check{\delta}_{n}}{z_{n}}$,
(iii) $\breve{w}_{B} \bullet q \breve{z}_{B}=\frac{\check{\delta}_{n} \bullet q \hat{\delta}_{n}}{z_{n} \bullet q y_{n}}$,
(iv) $D^{k} \breve{w}_{B}=\frac{D^{k} \breve{\delta}_{n}}{z_{n}}$, and
(v) $\breve{w}_{B} \bullet q \check{\delta}=\frac{\check{\delta}_{n} \bullet q \check{\delta}}{z_{n}}$,

where $k \in \mathbb{R}, \beta \in \mathbb{C}$, and $D^{k} \breve{w}_{B}$ is the $k$ th derivative of $\breve{w}_{B}$ and $\check{\delta} \in L_{q}^{1}$.

Definition 16 For $n=1,2,3, \ldots$ and $\breve{w}_{B, n}, \breve{w}_{B} \in B_{q}^{2}$, the sequence $\left(\breve{w}_{B, n}\right)$ is said to be $\delta_{q}$ convergent to $\breve{w}_{B}$, denoted by $\delta_{q}-\lim _{n \rightarrow \infty} \breve{w}_{B, n}=\breve{w}_{B}$, provided there can be found a delta sequence $\left(z_{n}\right), z_{n}=S_{q} x_{n}, n \in \mathbb{N}$, such that

(a) $\left(\breve{w}_{B, n} \bullet^{q} z_{k}\right),\left(\breve{w}_{B} \bullet^{q} z_{k}\right)$ in $L_{q}^{1}$ for all $n, k \in \mathbb{N}$;

(b) $\lim _{n \rightarrow \infty} \breve{w}_{B, n} \bullet^{q} z_{k}=\breve{w}_{B} \bullet^{q} z_{k}$ in $L_{q}^{1}$ for every $k \in \mathbb{N}$.

Definition 17 For $n=1,2,3, \ldots$ and $\breve{w}_{B, n}, \breve{w}_{B} \in B_{q}^{2}$, the sequence $\left(\breve{w}_{B, n}\right)$ is said to be $\Delta_{q}^{S_{q}}$ convergent to $\breve{w}_{B}$, denoted by $\Delta_{q}-\lim _{n \rightarrow \infty} \breve{w}_{B, n}=\breve{w}_{B}$, provided there can be found a delta sequence $\left(z_{n}\right)$ such that

(a) $\left(\breve{w}_{B, n}-\breve{w}_{B}\right) \bullet{ }^{q} z_{n} \in L_{q}^{1}(\forall n \in \mathbb{N})$;

(b) $\lim _{n \rightarrow \infty}\left(\breve{w}_{B, n}-\breve{w}_{B}\right) \bullet^{q} z_{n}=0$ in $L_{q}^{1}$.

Remark 18 Let $\check{\delta} \in L_{q}^{1}$ and $\left(z_{n}\right) \in \Delta_{q}^{S_{q}}, z_{n}=S_{q} x_{n}, n \in \mathbb{N}$, be fixed. Then the mapping

$$
\check{\delta} \rightarrow \breve{w}_{B}
$$

where $\breve{w}_{B}=\frac{\check{\delta}_{\bullet} q_{z_{n}}}{z_{n}}$ is an injective mapping from $L_{q}^{1}$ into $B_{q}^{2}$.

Therefore, it can be easily checked that $L_{q}^{1}$ may be identified as a subspace of $B_{q}^{2}$.

Remark 19 Let $\left(z_{n}\right) \in \Delta_{q}^{S_{q}}, z_{n}=S_{q} x_{n}, n \in \mathbb{N}$. Then if $\check{\delta}_{n} \rightarrow \check{\delta}$ in $L_{q}^{1}$ as $n \rightarrow \infty$, then, for all $k \in \mathbb{N}$,

$$
\check{\delta}_{n} \bullet{ }^{q} z_{k} \rightarrow \check{\delta} \bullet{ }^{q} z_{k}
$$

as $n \rightarrow \infty$. That is, $\breve{w}_{B, n} \rightarrow \breve{w}_{B}$ in $B_{q}^{2}$ as $n \rightarrow \infty$.

The above remark states the following.

Theorem 20 The mapping $\psi \rightarrow \breve{w}_{B}, \breve{w}_{B}=\frac{\check{\delta}_{\bullet} q_{z_{n}}}{z_{n}}$, is a continuous $q$-embedding of the space $L_{q}^{1}$ into the space $B_{q}^{2}$. 


\section{The $q$-Sumudu transform of generalized $q$-theory}

Definition 21 Let $\frac{\check{\delta}_{n}}{x_{n}}$ be a $q$-Boehmian in the space $B_{q}^{1}$. Then we define the $q$-Sumudu transform of $\frac{\check{\delta}_{n}}{x_{n}}$ as follows:

$$
S_{q}^{1} \frac{\check{\delta}_{n}}{x_{n}}=\frac{S_{q} \check{\delta}_{n}}{S_{q} x_{n}} \quad \text { for all }\left(\check{\delta}_{n}\right) \in L_{q}^{1} \text { and }\left(x_{n}\right) \in \Delta_{q} \text {. }
$$

It is clear that $S_{q}^{1} \check{\check{\delta}}_{n}$ belongs to $B_{q}^{2}$ as $\left(S_{q} \check{\delta}_{n}\right)$ and $\left(S_{q} x_{n}\right)$ are elements of the spaces $L_{q}^{1}$ and $\Delta_{q}^{S_{q}}$, respectively. The linearity of $S_{q}^{1}$ follows by easy techniques.

Theorem 22 The operator $S_{q}^{1}: B_{q}^{1} \rightarrow B_{q}^{2}$ is q-sequentially continuous, i.e., if $\Delta_{q}-$ $\lim _{n \rightarrow \infty} \breve{w}_{B, n}=\breve{w}_{B}$ in $B_{q}^{1}$, then

$$
\Delta_{q}-\lim _{n \rightarrow \infty} S_{q}^{1} \breve{w}_{B, n}=S_{q}^{1} \breve{w}_{B} \quad \text { in } B_{q}^{2}
$$

Proof Let $\Delta_{q}-\lim _{n \rightarrow \infty} \breve{w}_{B, n}=\breve{w}_{B}$ in $B_{q}^{1}$, then there is $\left(x_{n}\right)$ in $\Delta_{q}$ such that $\Delta_{q}-$ $\lim _{n \rightarrow \infty}\left(\breve{w}_{B, n}-\breve{w}_{B}\right) *^{q} x_{n}=0$ in $B_{q}^{1}$. The continuity of the integral operator gives

$$
\Delta_{q}^{S_{q}}-\lim _{n \rightarrow \infty} S_{q}^{1}\left(\left(\breve{w}_{B, n}-\breve{w}_{B}\right) *{ }^{q} x_{n}\right)=\Delta_{q}^{S_{q}}-\lim _{n \rightarrow \infty}\left(\left(S_{q}^{1} \breve{w}_{B, n}-S_{q}^{1} \breve{w}_{B}\right) \bullet{ }^{q} S_{q} x_{n}\right)=0 .
$$

Thus, we have $\Delta_{q}^{S_{q}}-\lim _{n \rightarrow \infty} S_{q}^{1} \breve{w}_{B, n}=S_{q}^{1} \breve{w}_{B}$ in $B_{q}^{2}$.

This finishes the proof of the theorem.

Theorem $23 S_{q}^{1}: B_{q}^{1} \rightarrow B_{q}^{2}$ is one-one, onto, continuous with respect to $\delta_{q}$ and $\Delta_{q^{-}}$ convergence and consists with the classical operator $S_{q}$.

Proof Proofs of the parts that $S_{q}^{1}$ is one-one, onto, continuous with respect to $\delta_{q}$ and $\Delta_{q^{-}}$ convergence are analogous to those given in the literature. To prove that $S_{q}^{1}$ consists with the classical operator $S_{q}^{1}$, let $\hat{\delta} \in L_{q}^{1}$ and let $\frac{\hat{\delta}_{*} q_{z_{n}}}{z_{n}}$ be its representative in $B_{q}^{2}$ for all $z_{n}=S_{q} x_{n}$, $\left(x_{n}\right) \in \Delta_{q}$. Clearly, for all $n \in \mathbb{N},\left(z_{n}\right)$ is independent of the representative. Hence, by the convolution theorem we get

$$
S_{q}^{1} \frac{\hat{\delta} *^{q} z_{n}}{z_{n}}=\frac{S_{q} \hat{\delta} \bullet \bullet^{q} S_{q} x_{n}}{S_{q} x_{n}}=S_{q} \hat{\delta} \bullet{ }^{q} S_{q}^{1} \frac{x_{n}}{x_{n}} .
$$

That is, $\frac{S_{q} \hat{\delta}_{\bullet} S_{q} x_{n}}{S_{q} x_{n}}=\frac{S_{q} \hat{\delta}_{\bullet} q_{n}}{z_{n}}$ is the representative of $S_{q} \hat{\delta}$ in the space $L_{q}^{1}$. The proof is, therefore, finished.

We introduce the transform inversion formula as follows.

Definition 24 We define the inverse integral operator of $S_{q}^{1}$ of a $q$-Boehmian $\frac{S_{q} \check{\check{q}}_{n}}{z_{n}}$ in $B_{q}^{2}$ as a $q$-Boehmian in $B_{q}^{1}$ defined by

$$
S_{q}^{-1} \frac{S_{q} \check{\delta}_{n}}{z_{n}}=\frac{\check{\delta}_{n}}{x_{n}}
$$

where $z_{n}=S_{q} x_{n},\left(x_{n}\right)$ is a delta sequence in $\Delta_{q}$, and $\left(\check{\delta}_{n}\right)$ is a sequence of integrable functions in $L_{q}^{1}$. 
Theorem 25 Let $\frac{S_{q} \check{\delta}_{n}}{z_{n}}$ be a q-Boehmian in $B_{q}^{2}, z_{n}=S_{q} x_{n},\left(x_{n}\right) \in \Delta_{q}$, and $\hat{\delta} \in L_{q}^{1}$. Then we have

$$
S_{q}^{1}\left(\frac{\check{\delta}_{n}}{x_{n}} *^{q} \hat{\delta}\right)=\frac{S_{q} \check{\delta}_{n}}{z_{n}} \bullet^{q} \hat{\delta} \quad \text { and } \quad S_{q}^{-1}\left(\frac{S_{q} \check{\delta}_{n}}{z_{n}} \bullet^{q} \hat{\delta}\right)=\frac{\check{\delta}_{n}}{x_{n}} *^{q} \hat{\delta}
$$

Proof Assume that $\frac{S_{q} \check{\delta}_{n}}{z_{n}}$ is a $q$-Boehmian in the space $B_{q}^{2}, z_{n}=S_{q} x_{n},\left(x_{n}\right) \in \Delta_{q}$, and $\hat{\delta} \in L_{q}^{1}$. Then, by using the convolution theorem, Definition 21, and Eq. (9), we have

$$
\begin{aligned}
S_{q}^{1}\left(\frac{\check{\delta}_{n}}{x_{n}} *^{q} \hat{\delta}\right) & =S_{q}^{1}\left(\frac{\check{\delta}_{n} *^{q} \hat{\delta}}{x_{n}}\right) \\
& =\frac{S_{q}\left(\check{\delta}_{n} *^{q} \hat{\delta}\right)}{S_{q} x_{n}} \\
& =\frac{x S_{q} \check{\delta}_{n}(x) S_{q} \hat{\delta}(x)}{S_{q} x_{n}} \\
& =\frac{S_{q} \check{\delta}_{n} \bullet^{q} \hat{\delta}}{S_{q} x_{n}} \\
& =\frac{S_{q} \check{\delta}_{n}}{S_{q} x_{n}} \bullet^{q} \hat{\delta} \\
& =\frac{S_{q} \check{\delta}_{n}}{z_{n}} \bullet^{q} \hat{\delta} .
\end{aligned}
$$

Similarly, by using the convolution theorem, Definition 24, and Eq. (9), we obtain

$$
\begin{aligned}
S_{q}^{-1}\left(\frac{S_{q} \check{\delta}_{n}}{z_{n}} \bullet^{q} \hat{\delta}\right) & =S_{q}^{-1} \frac{S_{q} \check{\delta}_{n} \bullet^{q} \hat{\delta}}{z_{n}} \\
& =S_{q}^{-1} \frac{x S_{q} \check{\delta}_{n}(x) S_{q} \hat{\delta}(x)}{z_{n}} \\
& =S_{q}^{-1} \frac{S_{q}\left(\check{\delta}_{n} *^{q} \hat{\delta}\right)}{S_{q} x_{n}} \\
& =\frac{\check{\delta}_{n} *^{q} \hat{\delta}}{x_{n}} \\
& =\frac{\check{\delta}_{n}}{x_{n}} *^{q} \hat{\delta} .
\end{aligned}
$$

This completely finishes the proof of the theorem.

\section{Conclusion}

This paper could be an evolution of idea. It gives an extension to a set of $q$-integrable functions to a set of $q$-integrable $q$-generalized functions. It verifies that the $q$-analysis of this paper generalizes the $q$-analysis followed by Albayrak et al. 2013. Moreover, this paper has also shown that the generalized $q$-Sumudu transform and its $q$-inversion formula are well-defined mappings possessing properties alike to the classical properties of their corresponding classical versions. 


\section{Acknowledgements}

The author would like to thank his parents and friends for their support.

\section{Funding}

No funding sources to be declared.

\section{Availability of data and materials}

Please contact the author for data requests.

\section{Competing interests}

The author declares that they have no competing interests.

\section{Authors' contributions}

The authors contributed equally and significantly in writing this paper. All authors read and approved the final manuscript.

\section{Publisher's Note}

Springer Nature remains neutral with regard to jurisdictional claims in published maps and institutional affiliations.

Received: 28 August 2020 Accepted: 26 November 2020 Published online: 07 January 2021

\section{References}

1. Kritika, A.R., Purohit, S.D.: Solution of fractional kinetic equations associated with the $(p, q)$-Mathieu-type series. Discrete Dyn. Nat. Soc. 2020, 1-7 (2020)

2. Nisar, K.S.: Generalized Mittag-Leffler type function: fractional integrations and application to fractional kinetic equations. Front. Phys. 8, 1-7 (2020)

3. Jackson, F.H.: On q-definite integrals. Q. J. Pure Appl. Math. 41, 193-203 (1910)

4. Ucar, F:: q-Sumudu transforms of $q$-analogues of Bessel functions. Sci. World J. 2014, 1-7 (2014)

5. Abdi, W.H.: On q-Laplace transforms. Proc. Natl. Acad. Sci., India 29, 389-408 (1961)

6. Albayrak, D., Purohit, S.D., Ucar, F.: On q-Sumudu transforms of certain q-polynomials. Filomat 27(2), 413-429 (2013)

7. Hahn, W.: Beitrage Zur Theorie Der Heineschen Reihen, die 24 Integrale der hypergeometrischen q-diferenzengleichung, das q-Analog on der Laplace transformation. Math. Nachr. 2, 340-379 (1949)

8. Al-Omari, S.K.: On $q$-analogues of the natural transform of certain $q$-Bessel functions and some application. Filomat 31(9), 2587-2598 (2017)

9. Fitouhi, A., Bettaibi, N.: Applications of the Mellin transform in quantum calculus. J. Math. Anal. Appl. 328, 518-534 (2007)

10. Exton, H.: A basic analogue of the Bessel-Clifford equation. Jñānābha 8, 49-56 (1978)

11. Shanoja, R.N., Haubold, H.J.: On the q-Laplace transform and related special functions. Axioms 5, 24 (2016)

12. Al-Omari, S.K.Q.: On $q$-analogues of the Mangontarum transform for certain $q$-Bessel functions and some application. J. King Saud Univ., Sci. 28(4), 375-379 (2016)

13. Albayrak, D., Purohit, S.D., Ucar, F.: On q-analogues of Sumudu transform. An. Ştiinţ. Univ. 'Ovidius' Constanţa 21(1), 239-260 (2013)

14. Al-Omari, S.K.Q.: On q-analogues of Mangontarum transform of some polynomials and certain class of H-functions Nonlinear Stud. 23(1), 51-61 (2016)

15. Meena, S., Bhatter, S., Jangid, K., Purohit, S.D.: Certain integral transforms concerning the product of family of polynomials and generalized incomplete functions. Moroccan J. Pure Appl. Anal. 6(2), 243-254 (2020)

16. Albayrak, D., Purohit, S.D., Ucar, F.: On $q$-Laplace and $q$-Sumudu transforms of a product of generalized q-Bessel functions. Math. Eng. Sci. Aerosp. 11(2), 355-369 (2020)

17. Albayrak, D., Purohit, S.D., Ucar, F.: Certain inversion and representation formulas for $q$-Sumudu transforms. Hacet. J. Math. Stat. 43(5), 699-713 (2014)

18. Nisar, K.S., Shaikh, A., Rahman, G., Kumar, D.: Solution of fractional kinetic equations involving class of functions and Sumudu transform. Adv. Differ. Equ. 2020, 39 (2020)

19. Purohit, S.D., Ucar, F.: An application of $q$-Sumudu transform for fractional $q$-kinetic equation. Turk. J. Math. 42 , 726-734 (2018)

20. Boehme, T.K.: The support of Mikusinski operators. Trans. Am. Math. Soc. 176, 319-334 (1973)

21. Belgacem, F.B.M., Karaballi, A.A., Kalla, S.: Analytical investigations of the Sumudu transform and applications to integral production equations. Math. Probl. Eng. 2003(3), 103-118 (2003)

22. Al-Omari, S.K.Q.: Hartley transforms on certain space of generalized functions. Georgian Math. J. 20(3), 415-426 (2013)

23. Al-Omari, S.K.Q., Baleanu, D.: On generalized space of quaternions and its application to a class of Mellin transforms. J. Nonlinear Sci. Appl. 9, 3898-3908 (2016)

24. Al-Omari, S.K.Q.: A fractional Fourier integral operator and its extension to classes of function spaces. Adv. Differ. Equ. 195, 1-9 (2018)

25. Al-Omari, S.K.Q.: Some estimate of a generalized Bessel-Struve transform on certain space of generalized functions. Ukr. Math. J. 69(9), 1155-1165 (2017)

26. Al-Omari, S.K.Q., Baleanu, D.: On the generalized Stieltjes transform of Fox's kernel function and its properties in the space of generalized functions. J. Comput. Anal. Appl. 23, 108-118 (2017)

27. Al-Omari, S.K.Q., Agarwal, P., Choi, J.: Real covering of the generalized Hankel-Clifford transform of fox kernel type of a class of Boehmians. Bull. Korean Math. Soc. 52(5), 1607-1619 (2015)

28. Al-Omari, S.K.Q., Baleanu, D.: Quaternion Fourier integral operators for spaces of generalized quaternions. Math. Methods Appl. Sci. 41, 9477-9484 (2018) 
29. Al-Omari, S.K.Q.: On some Whittaker transform of a special function kernel for a class of generalized functions. Nonlinear Stud. 26(2), 435-443 (2019)

30. Al-Omari, S.K.Q.: On Some Variant of a Whittaker integral operator and its representative in a class of square integrable Boehmians. Boletim da Sociedade Paranadade de Matematics 38(1) (2020)

31. Al-Omari, S.K.Q.: Some remarks on short-time Fourier integral operators and classes of rapidly decaying functions Math. Methods Appl. Sci. 41, 1-8 (2018)

32. Belgacem, B.M., Karaballi, A.: Sumudu transform fundamental properties investigations and applications. J. Appl. Math. Stoch. Anal. 2006, Article ID 91083 (2006)

Submit your manuscript to a SpringerOpen ${ }^{\odot}$ journal and benefit from:

- Convenient online submission

Rigorous peer review

- Open access: articles freely available online

- High visibility within the field

- Retaining the copyright to your article

Submit your next manuscript at $\gg$ springeropen.com 